Interviews 


\section{An Interview with Shyam Ranganathan}

ABDUL HALIM

Shyam Ranganathan (hereafter SR) teaches introduction to philosophy, critical reasoning, ethics, political philosophy, Asian philosophy, the philosophy of religion and the philosophy of language in the York University, Toronto. He has a research specialization in a Non-Western tradition of philosophy -namely South Asian philosophy, especially Indian moral philosophy. Abdul Halim (hereafter AH) is an assistant editor of the Translation Today who interviews Shyam Ranganathan.

AH: Translation and Interpreting Studies have made significant advances ever since they became formal disciplines. How do you see the current trends in Translation \& Interpreting Studies?

SR: Since I first started working on this in my dissertation (15 years ago), I started noticing a trend in academic views about translation and interpretation that were not restricted to the interdisciplinary fields of Translation and Interpreting Studies. I found it in the Analytic philosophy literature, the Continental philosophy literature, the Translation Studies literature, as well as the writings and assumptions of Indologists who claimed to be studying Indian philosophy. I now think that this trend is just as old as the Western tradition itself. This is the trend of identifying propositional contentthe stuff to be preserved in translation - as linguistic meaning. This is often associated with the linguistic turn in recent Continental and Analytic philosophy but it goes back to the Greek idea of logos: one word for thought, opinion, reason and word. This is the most basic commitment of the Western 
tradition and something that started to jump out of the literature at me as I read more and more of it. This tradition has a problem. The meanings of our languages are historically varied owing to their respective histories. This is unavoidable. Assume the orthodox view in the literature that the literal meaning of an expression in a language is its systematic or basic use or role in the language. Even syntactic differences across languages will produce semantic differences on this account. If thought and what is to be preserved in translation is linguistic meaning, then the mere fact of linguistic differences dooms translation for we have no guarantee that languages will be semantically alike enough to facilitate translation on this account. And there is a paradox: the more dissimilar languages are, and the more we require translation, the less we are likely to be able to translate on this account. The problem here just is the idea that it is the linguistic meaning that translation is supposed to be devoted to, as though accuracy in translation is about preserving linguistic meaning. The one positive matter that I note about Translation and Interpreting Studies literature is an often disciplined distinction between translation and interpretation. Philosophers tend not to draw this important distinction. But clearly there is a difference. To translate is to preserve something deep (thought) through changes in semiotic resources so that the outcome has to be the same as the original-in the deep propositional sense-though superficially it is not. Interpretation is about explaining what someone else says (hence, simultaneous interpretation is not translation). So this is one area where people outside of philosophy in Translation and Interpretation Studies working on these issues are leaps and bounds ahead of conventional Western philosophers who, whether in the Analytic or Continental traditions, routinely confuse interpretation and translation. But thinking about the difference is useful for it helps us pry apart the semantic preserving project of 
translation with the explanatory function of interpretation. A good translation does not explain the original: it is rather the same work, composed with differing materials. A good interpretation explains the original, and it might even do so with the same materials.

AH: Two of your publications "Philosophy of Language, Translation Theory and a Third Way in Semantics" and "An Archimedean Point for Philosophy" and your own doctoral thesis "Translating Evaluative Discourse: The Semantics of Thick and Thin Concepts" have dialectically investigated all the parameters set by the philosophers of language and translation theorists in Translation Studies. Could you please explicate the gap between the theoretical postulations and the practical act of translation?

SR: Well, I think the contrast is probably between good theory and bad theory, for if one employs good theory then one's practice will be good, and if one employs bad theory, then one's practice will be bad. But there is a way in which I can understand that there is a gap between theory (conventional theory) and practice, in so far as actual translators (and certainly the good ones) tend not to follow the advice of most philosophers and theorists of translation. The dominant position in the Western tradition is that meaning and thought is linguistic so to translate a text accurately requires that we pair up words and sentences across languages with the same meaning, for this is the only way to preserve the thoughts expressed in the original text. And given the historical reality of linguistic difference, this is impossible exactly when we need it. Indeed, we can even identify a paradox that arises on the basis of this account of translation: the more similar languages are, the more easily translatable they are, the less we require translation for the more inter-intelligible they are. The 
less similar languages, the more require translation for the less inter-intelligible they are, but the less easily are such languages translatable. The problem it seems to me is the expectation that we should be proceeding by preserving linguistic meaning. This is exactly what good translators do not worry about. They re-create a work in a new medium, and just as we would recreate a sculpture or painting with a new medium, we do not judge the accuracy of the resulting product in terms of its one to one correspondence on the microscopic level. A sculpture made of pebbles and one made of pasta shells can express the same form and even appear identical holistically, but that is macroscopic, and it is not reducible to the similitude of corresponding parts. So good translators are after that total recreation, and to do that, you have to give up the idea that we translate by matching words and sentences, as though reproducing a sculpture with pasta means that we have to match pieces of pasta with pieces of stone in the original. But this entails something important: translation is not about linguistics. It is not at all about understanding the similarity of words and sentences across languages. You have to be able to discern the form of the original text and have the artistic facility to recreate this form with differing resources. There's such a thing as getting this re-creation right and wrong: that's objective, macroscopically. It is a very nerdy idea of translation that suggests that it's a kind of been counting, where one has to be worried about the minutia.

AH: As your writings reflect, language is not the primary bearer of meaning and in support of your argument you have proposed a text-type conception of semantics. How would you reflect on a translation theory which could address the issues of translation encompassing all the academic disciplines? And what would be the semantic expansion of meaning making process in translation? 
SR: When I originally formulated my argument I thought the idea of a text-type was perfect. However, it only puts off a question: how do we individuate types? I used to claim that they were merely institutional practices and this is true, but this invites the challenge that there can be differing institutions for every type and evidence of this is that within a discipline, parties disagree and each party to the disagreement has their own idea of what the type is. This problem can be solved by identifying the type with disciplinarity. And this is actually an Indian theory: yoga. It was a pure coincidence that as I was working on my dissertation on translation theory I took up the task of translating the Yoga Sütra. And then I had to work on the Yoga Sütra as a historian of philosophy for another several years before I started to understand its relevance. But the idea of yoga in the Indian tradition and especially in Patanjali is the idea of a practice that we can undertake from differing perspectives. So we individuate the yoga then as this continuity as we change our position in the world. Then, differing practices will allow us to triangulate on differing objects of inquiry. This is why we distinguish disciplines in higher learning, such as the difference between the empirical sciences, mathematics, philosophy, literature, history etc. In each case we have a differing kind of practice that makes tracking common objects from differing (theoretical) vantages possible. This is why disciplinarity is the foundation of knowledge: it allows us to conduct research into objects of interest from competing vantages. It follows from this that disagreements within a discipline are par for course, and not evidence that we have more than one discipline at play. Really the discipline is what makes the disagreement possible in the first place for it allows us to take up contrary positions relative to objects of controversy. If we were to really follow the Western tradition and identify the topic of inquiry as logos, linguistics would be the only discipline and every kind of 
research would be some version of linguistics. Some philosophers have fantasized about this. Hilary Putnam did this in his famous "Meaning of Meaning"- he claimed that every discipline represents the division of linguistic labour. Physicists would be trying to figure out what our physical terms mean. Biologists would be trying to figure out what our biological terms mean, so on and so forth. But it is implausible. It is implausible because we can conduct the same inquiry (physics, literary criticism etc.,) as we change the language we employ: one can do physics in English and in Hindi and in Mandarin. We can do literary criticism in these languages too. These are not the same languages and they are three differing languages. Yet, the topic of investigation does not change merely because the language we use to talk about it changes. Rather, we know we are in the realm of a discipline because we use differing linguistic and cultural resources to talk about the same thing. In other words, disciplinarity allows us to transcend the provincial, parochialism of language and culture, and engage in knowing (jñāna). But if it were mere linguistics, then research into English language physical terms would be different from research into Mandarin language physical terms: change the language and one changes the topic. So English physics would be different from Mandarin physics. What allows for this continuity of research across languages is the discipline. So we ultimately have to individuate the text-type by the discipline and this allows us to identify what is essential and distinctive about types. So in other words, the disagreement within disciplines is evidence of the underlying common text type. But the text type is nothing but a semantic approach to what is basically a matter of practice: discipline. One of the implications of this line is that we must and should draw a sharp line between translation and localization. Translation concerns disciplinarity. Localization does not. 
So far as the meaning making process is concerned we use meaningful devices for some text-type theoretic purpose, and this use is a textual meaning. So translation then is not the process of creating meaning so much as using differing resources to preserve textual meaning. Good translation preserves this textual meaning and is hence uncreative. It may seem novel to the target audience but that is an illusion that arises from them taking their vantage too seriously. If it's accurate it's not new: it preserves the original meaning. But then all translators should not look upon their task as making something new. However, if the idea of "semantic expansion" could mean something like the introduction of a new idea or theory into a target culture, then translation-good translation - can achieve this as a matter of course.

AH: While most of the theorists have described the problem of translation from functionalist perspectives that is useful mainly for literary translation, what approach would you like to propose for the translators who take up all kinds of translation? And what should be semantic aspects of text-type features for determinate translation and how non-text-type features could be preserved in translation?

SR: Functionalism I take it is the idea that the meaning of what is said is the effect it has on its audience, and functionalism in translation is the idea that an accurate translation is equality in effect to the original, though it may be different literally. I do think this is implausible. The people who make a case for such a theory do not translate philosophy, logic, mathematics, or science. They usually base their case on poetry, and literature. It is plausible to think that the accuracy of a poetic translation is to be judged by the similitude of emotional response to the reader relative to the original and translation. It is implausible —absolutely implausible - to 
employ the same standard for the translation of philosophy, mathematics or science. And those who insist upon functionalism never use examples from philosophy, science or mathematics: they typically stick to examples from literature.

But there is a bigger picture.

In every case, the translator is faced with a choice: what type should I employ in translation? I say this is a choice because texts themselves are ambiguous, and as the process of translation is one of preserving an integral meaning to a work in translation (usually the propositional content, which seems to me to be the same as the holistic significance) you have to choose. And the results will be uneven: sometimes you get it right, and sometimes you do not. If I try to translate excellent poetry as mathematics, I likely will end up with nothing that counts as an accurate translation for the original text will likely be devoid of math. Yet I have to choose a text-type in translation because I have to choose what is to be the priority in the process of translation (recreating the form of the original with differing materials). When translating Plato, I can choose to read and translate him as a dramatist first, or a philosopher first. If I choose drama as my type, I treat the philosophical elements as subsidiary to the dramatic aspects, and the resulting translation could succeed if it creates a target text with the same dramatic virtues that subordinate philosophical virtues in the same way. But what translated bit of philosophy serves this dramatic purpose may not at all be the same as the philosophical arguments in the original. For instance, if the philosophy in the original served to articulate some conservative position and the conservatism was somehow essential to appreciating the dramatic components, then a good dramatic translation will have to rely upon some conservative philosophy from the target culture to serve the same purpose- 
and this may be very different philosophy from what is found in the source culture. If I choose to translate Plato as philosophy, then I treat the dramatic components of his dialogues as supporting his philosophical aims, which means that as I translate these dramatic components, I will subordinate them to the philosophy in the same way, but this might mean that the drama in the target text looks different than the drama in the source text. If a pun or joke is essential to making a point in an argument, the translated joke or pun has to make sense to the target audience, and this may be a very different joke or pun from the original.

But in each case, I am avoiding the functionalist approach for I am abandoning the idea that translatable content is to be measured purely in terms of its effect on their audiences. I am choosing a type, and then subordinating other features of the texts to the main type.

AH: Since the publication of James S. Holmes' article "The Name and Nature of Translation Studies" the discipline of Translation Studies has taken mainly two recognizable turns namely linguistic and cultural. How far these two trends in translation have contributed to address the actual problem of translation? Do you see any (in) adequacies in them?

SR: I am not entirely sure I understand the difference. I know that people draw a distinction between linguistic and cultural approaches. I suppose in some sense the linguistic is the more traditional approach where translatable content is defined as literal meaning (the systematic or basic role of an expression in a language), while the cultural approach is a bit more like the functionalist approach. This time we are invited to be reflective about the effect that translation has. But as this approach reduces the significance of a text to its cultural effect, 
it is not so different from the linguistic approach as the linguistic approach also reduces the significance of a text to a crucial cultural factor: language. And hence they share an inadequacy of trying to understand translation by way of matters that are peculiar to cultures. The worse pressure that this creates is to look for similarities where there are none. For if we have to identify the translatable content of a text with some cultural factor, then we are pressurized to find something similar in the target culture that can function as the translation and the result is confabulation. Really, the problem is that these approaches do not specify an independent measure of translational success: in each case, the standard is reduced to the vary languages or cultures that we are trying to mediate by translation.

AH: Analytical and continental philosophers of language are sceptical in describing the phenomena of translation. They observe that translation is indeterminate and incommensurable. Nevertheless, translation is taking place all around the globe. What ideological factors may be motivating it?

SR: These philosophers are faithful to the Western tradition, which going back to the Greek idea of logos, holds that thought content is linguistic. So if you really believe that, and you are also aware of the reality of linguistic difference, then you have no choice but to decide that translation is beset by problems of incommensurability or problems of indeterminacy. Just to be clear, I take it that incommensurability is the problem of finding one to one correlates across languages, and differing languages will hence be to varying degrees, incommensurable. "Indeterminacy" is a term that Quine made famous and in his case it meant that even when we have all the relevant empirical data we may be unable to decide between alternative translations, where the 
alternative translations are not translations of each other. But this too is assured on the linguistic paradigm for we are speaking about trying to match up incommensurable thingsthe meaning of expressions defined by their role in their respective languages. So indeed, the empirical data is not going to help and we may have good reason for choosing translations that are not themselves translations of each other.

AH: You specialize in analytical philosophy but you are writing from non-Western perspectives. Could you offer some ideas about the Western notion of translation vis-à-vis anuvaad, the Indian tradition including the boundary of translation terminologies used in both the traditions and cultures?

SR: So this is a question I find difficult to answer because I have never thought about translation as a question of terminology. So I have never really paid much attention to what Indians might have called what Westeners call translation. I have been far more impressed by the importance of Indian philosophy and Indian philosophies to solving problems. So for instance, the distinction between Two Truths, in the Indian tradition, seems pertinent to translation. One kind of truth, the conventional truth, would apply to categories of language and cultural distinctions, while the Ultimate Truth pertains to the reality of the matter. Good translations preserve the Ultimate Truth of a text, so to speak, while trading Conventional Truths. That is a good way to approximate the issue of translation-far better than trying to understand it as preserving culture or linguistic meaning. Another Indian idea that I think is essential to understanding translation is the idea of disciplinarity, or yoga, as I noted earlier. It is perhaps easier for Indian philosophers to make sense of translation for they have wrestled with the idea of continuity despite change (often with respect to questions of personal identity) than Western 
philosophers have. Indian philosophers have often accepted the reality of change and have asked the question: what makes me the same over time? That's basic to translation. So to the extent that Indian ideas of continuity of identity allow for change, they allow us a way to think about translation. A healthy life, where we preserve ourselves despite change is a kind of exercise in translation. Buddhists hold this is impossible in the big picture: dependency and change wins. But I think the idea of disciplinarity and Yoga associated with the "Hindu" tradition allows something else: for sure it's possible and it has to do with disciplinarity. I am the same person I used to be not because microscopically I am the same now as I used to be, but relative to the practice of being me, I am the same, macroscopically. Now existentially the problem for ethics is that we don't usually succeed: at some point it seems that by any account of the practice of me, I've changed beyond recognition and at that point I'm dead. Yet, this is not an objection to Yoga for so long as I have a criterion for what it is to be the same macroscopically while I change microscopically I have a way to adjudicate good translations of me over time relative to bad translations.

AH: Many scholars have interpreted translation as a site of knowledge creation and dissemination. How do you reflect upon this view?

SR: Well, when we link translation to text types and these to disciplines, then indeed, translation is about the creation and dissemination of knowledge in so far as disciplines are sites of knowledge creation and dissemination.

AH: Every translation has problems of its own, and a good translation depends on the correct understanding of the subject matter. What would be the correct methods which 
could be generalized to all kinds of translation?

SR: Great question! Again, I think I have already answered the question. (A) We have to first choose a type, and then (B) subordinate the other elements of a text to the type. This allows us an understanding of the original text relative to the type, which we may call the work. And then (C) we reproduce a work that is equivalent to the original text with new target resources.

AH: Like there is no manual for guiding a writer, there is an overall absence of a manual to guide the translators at various stages of translating a text. All these depend on the correct understanding of a translator/writer of the issues concerned. How can it be achieved and will there be possibly a manifesto of translation like the Communist Manifesto?

SR: I think that a manual could be written. You would have to specify the various steps such as (A), (B) and (C) above and perhaps address common confusions. But this manual would not be the Quinian type envisioned in Word and Object, which is a kind of concordance that will allow anyone to translate a text, even if they do not understand the target, source languages, and even if they do not appreciate the relevant type. 